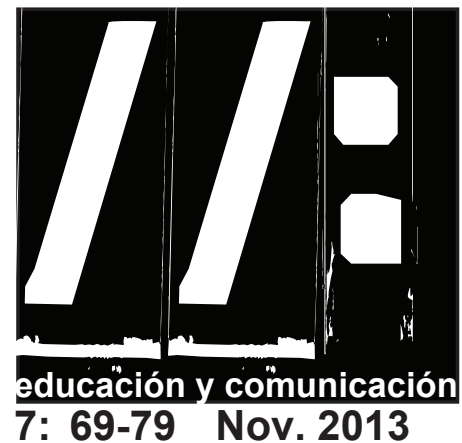

7: 69-79 Nov. 2013

\section{EDUCACIÓN INTERCULTURAL PARA El dESARROLLO PERSONAL Y COMUNITARIO}

\section{Intercultural Education For Personal and Communitry Development}

\author{
Eduardo S. Vila Merino \\ Víctor M. Martín Solbes \\ Facultad de Ciencias de la Educación \\ Universidad de Málaga (España) \\ E.mail: eduardo@uma.es \\ E.mail: victorsolbes@uma.es
}

Resumen:

No tiene sentido hablar de educación si no incluimos la dimensión intercultural en nuestras concepciones, reflexiones y acciones. En caso contrario nos referiremos en esencia a procesos formativos, adoctrinadores, disciplinarios, pero no eminentemente educativos; por tanto, una consecuencia lógica de esta premisa sería entender que toda política educativa y de desarrollo de las personas y las comunidades, debería estar impregnada de interculturalidad. Por ello vamos a abordar cuestiones relacionadas con la política educativa, revindicando coherencia a la hora de posicionarnos respecto a la elaboración de las políticas educativas interculturales. Para ello partiremos, en primer lugar, de unos principios necesarios para la reflexión de la interculturalidad desde la alteridad, como prerrequisito del desarrollo y la investigación educativa, para después ir introduciendo algunos elementos pedagógicos desde el análisis de las confluencias que, desde la interculturalidad, deben vincularse con nuestras sociedades, como es el fenómeno migratorio y sus consecuencias socioeducativas.

Palabras clave: Educación intercultural, derechos, desarrollo

\begin{abstract}
Summary:
There is no point speaking about education if we do not include intercultural dimensions in our conceptual, reflexive and active frameworks. On the contrary, we would be referring to formative processes, indoctrinating, disciplinary, but not strictly educative, ad for this reason a logical conclusion to our premise is that all educative policies should have an intercultural basis. In this light, we shall pose questions related to educative policies, and we shall demand ideological coherence when elaborating intercultural educative policies. For this reason, we shall set out to establish one of the principal methods necessary to reflect on interculturality from the perspective of otherness. This is a prerequisite for the development of educative research, so as to later introduce pedagogical elements of analysis that take into consideration interculturality. Therefore, themes such as migration and it socio-educative consequences must form a part of society.

Key words: Intercutural education, rights, development.
\end{abstract}

Recibido: 04-07-2013 / Revisado: 31-07-2013 / Aceptado: 20-08-2013 / Publicado: 01-11-2013

https://dx.doi.org/10.25267/Hachetetepe.2013.v2.i7.7 


\section{II: Educación y Comunicación para el Desarrollo}

\section{Políticas educativas interculturales y desarrollo de las personas y las comunidades}

$\mathrm{L}$ as políticas educativas, como ejes referenciales de las instituciones educativas y del sistema social que generan, de creciente complejidad en sus dinámicas y estructuras, han tenido una evolución histórica ligada a sus funciones de socialización, transmisión y re-elaboración de la cultura, así como a la cualificación personal y profesional de la ciudadanía. Pero, precisamente por esa evolución histórica (cultural, política, social y económicamente condicionada por una visión etnocéntrica), el sistema educativo se enfrenta actualmente a los 'nuevos' retos que le marca el economicismo hegemónico y a las 'viejas' exigencias sociales de igualdad y libertad a las que se debe desde su origen en el seno de la Modernidad, todo lo cual se hace más patente si cabe en el análisis de las cuestiones vinculadas al multiculturalismo cada vez más visible en nuestras sociedades. Así, en la actualidad nos encontramos inmersos en un modelo socioeconómico cuyas características principales suponen no sólo causas de desigualdades, reconocimiento de derechos y migraciones varias (locales y globales), sino también una lógica dominante del mundo actual que exige una mirada crítica como condición para un adecuado conocimiento de las dinámicas de acción social que trasciendan a las políticas neocoloniales que se disfrazan con términos como integración y concepciones impregnadas en el fondo de relativismo cultural.

En medio de todo esto se está produciendo un auge de todo lo vinculado con la diversidad cultural, fundamentalmente focalizado en el fenómeno migratorio, a través del que se abre un debate necesario a nivel social y cultural. Las personas migrantes suelen identificarse con lo distinto, diferente, extraño, ajeno, desconocido y, sobre todo, cuando esto se ve acompañado por condiciones socioeconómicas desfavorables, con lo no deseable e incluso peligroso. No olvidemos que la ignorancia y el miedo siempre han sido amigos de los prejuicios y la generalización, a la vez que enemigos de la diversidad, la justicia y la convivencia.

Desde esta perspectiva, consideramos un punto de partida ineludiblepara cualquierproyecto de desarrollo, tanto personal como social y de acción comunitaria (Oña, 2010), el que el tratamiento que se dé a estas cuestiones dentro de su epistemología y metodología tenga su primer referente en el derecho a la educación, a una educación no mercantilizada ni etnocéntrica, sino preñada de valores de respecto, reconocimiento y valoración a las diferencias culturales, sin caer en el 'romanticismo' ni el relativismo cultural. Por eso, es necesario, imprescindible diríamos, introducir estos nuevos debates en contextos multiculturales y educativamente diversos como el nuestro, todo ello sin dejar de hacer referencia a la cuestión de la legitimación de las desigualdades sociales, que sigue teniendo en el mito de la igualdad de oportunidades (que no es lo mismo que la igualdad de condiciones) su principal valedor, olvidándose el hecho de que para alcanzar el principio de igualdad debemos considerar nuestras diferencias y 'compensarlas' por medio de la equidad para alcanzar una real justicia educativa. Además, es necesario que visibilicemos la necesidad del bienestar emocional de los grupos migrantes que se traduce en el desarrollo de la autoestima o el sentido de estar protegido, ya que la ausencia de este 


\section{1/: Educación intercultural para el desarrollo}

bienestar puede originar patologías en el ámbito de lo psicológico (Bisquerra, 2000); esto nos iguala a todos, ya que no es algo que sólo se dé en los grupos migrantes, sino que todas las personas necesitamos de ese bienestar emocional para desarrollarnos equilibradamente y que nuestros entornos, también lo hagan.

En este contexto, la diversidad cultural que nos penetra e inunda, debe ser considerada como fuente de riqueza y entendimiento, a partir de la acción comunicativa con el otro y la otra, una acción que debe llevarse a cabo desde un marco referencial inclusivo donde tengan cabida nuestros respectivos universos simbólicos, partiendo desde categorías concretas factibles en la interacción y en facilitar espacios de convivencia, pero sin olvidar la vocación globalizadora de las mismas, otorgándole un papel protagonista para ello, a la ética, una vez establecida su contextualización en el mundo de las relaciones humanas, ya sean colectivas o simbólicas e instituidas, como diferencia Augé (1996), al analizar nuestro sentido social, y vinculadas con la pedagogía de la alteridad (Vila, 2005).

Si concretamos estas cuestiones y sus consecuencias para la configuración plural de nuestras sociedades y los retos que implican para las comunidades y la educación en todos sus niveles, desde la educación infantil a la educación de personas adultas y la educación superior, no podemos menos que reconocer su papel emergente como dimensión importante a analizar, no sólo en el campo de la política educativa sino también de la didáctica, la metodología y la manera de abordar los propios procesos de enseñanzaaprendizaje, abogando desde el primer momento por una perspectiva intercultural que perciba la riqueza que conlleva la creciente diversidad cultural y lingüística en nuestros entornos, ya que se trata de desarrollar a las personas y sus contextos, provocando procesos (Oña, op.cit).

Pero no podemos olvidar que ese pluralismo aludido supone a su vez un desafío desde esta percepción de la historia y la cultura como fuentes de una casi inagotable diversidad simbólica y de identidades individuales y colectivas. Es desde aquí que debemos partir de un concepto de cultura que nos sirva para dotar de sentido y significado el mundo que nos rodea, para aprender estrategias interpretativas de esa urdimbre a la que se refería Geertz (1991) en su clásica definición de cultura, para orientar, de este modo, nuestra acción a partir de dicha interpretación, generada no sólo desde las interacciones plurales del presente, sino también con el telón de fondo del horizonte del mundo o mundos de la vida. Por tanto, podríamos hablar de la cultura en términos de sistemas simbólicos que los individuos utilizan al construir los significados dentro del mundo de la vida, y de educación intercultural como conjunto de procesos educativos que valoran la interacción cultural desde el reconocimiento de derechos, el respeto a las diferencias, el sentido de pertenencia comunitaria y la generación de nuevas identidades a través de mundos de significados intersubjetivamente construidos.

Por otra parte, es necesario reconocer como pilar del desarrollo y la educación intercultural, el concepto de competencia cultural, entendida como un conjunto de comportamientos y actitudes que permite a las personas interactuar en contextos multiculturales de manera que incluya habilidades comunicativas, de representación y conocimiento en situaciones de diversidad cultural-lingüística. De esta manera, la 


\section{II: Educación y Comunicación para el Desarrollo}

competencia cultural se definiría por una especial sensibilidad hacia las diferencias, una conciencia social atenta a la ética y la igualdad en las relaciones y una visión de la alteridad como valor convivencial y referente educativo.

Por todo esto, es necesario tener presente que cuando intentamos analizar distintas maneras de afrontar esa pluralidad cultural desde el desarrollo de las personas y las comunidades, nos podemos encontrar con varios tipos de respuestas, que generalmente se presentan combinadas en la práctica social, que expresadas de forma sintética, serían:

- De negación, donde se realizan las políticas y prácticas sociales y educativas al margen de esta realidad multicultural, de manera homogénea.

- De marginación e invisibilización, que conlleva la percepción jerárquica de culturas y personas, segregando a aquellas que no se vean incluidas en los patrones hegemónicos y desarrollando tendencias asimilacionistas.

- De aceptación, donde la cultura mayoritaria acepta la existencia de las minoritarias, pero no pretende 'contaminarse' con las mismas.

- De integración, a partir de las cuales no sólo se percibe la existencia de la realidad multicultural sino que también se respetan las identidades de las diferentes culturas en un marco, no obstante diferenciado, en el que se acaba produciendo más una asimilación que un intercambio.

- De reconocimiento y respeto, observando e identificando las culturas desde sus peculiaridades, al mismo tiempo que implicándolas en la construcción de una cultura y una ciudadanía común, de manera que se valoren como positivas todas las culturas y se crea en el mestizaje como factor de enriquecimiento mutuo, atendiendo así a una mirada intercultural.

Así, cuando hablamos de interculturalidad lo hacemos también, inevitablemente, de la globalización y la exclusión como fenómenos sociales, puesto que en realidad no nos podemos referir a ambas como términos estáticos. Evidentemente no afecta de la misma manera la globalización a un niño o niña de nuestra ciudad o pueblo que a uno de algunos de los territorios más empobrecidos del planeta, o a personas de distintos barrios o contextos socioculturales, a las cuales no se les permite el mismo acceso, permanencia, expectativas y provecho del sistema educativo ni del mundo laboral. Además, debemos concebir la educación desde su función transformadora; en este sentido, Colom (2000) nos recuerda que educar es transformar, reconvertir, modificar, por lo que la educación intercultural y para el desarrollo de las personas y las comunidades, debe irradiar un halo pedagógico crítico, creativo, participativo y liberador de las personas.

\section{Tránsitos de teorías a prácticas interculturales}

Es necesario reflexionar sobre estas y otras cuestiones para poder construir discursos contrahegemónicos. Por ello es imprescindible asumir de facto una perspectiva intercultural que permita que los grupos y personas desfavorecidas y marginadas dejen de serlo y se sientan partícipes, escuchadas, valoradas y protagonistas de sus propias historias y las que colectivamente necesitamos construir desde el mestizaje intercultural. Así, desde esta óptica, y centrándonos en aspectos socioculturales y educativos, podemos establecer una serie de reflexiones que sinteticen y nos sirvan de guía para una conceptualización de la mirada intercultural 


\section{1/: Educación intercultural para el desarrollo}

en el ámbito de la educación para el desarrollo (Vila, 2012):

- La interculturalidad implica la lucha contra la exclusión desde la igualdad de diferencias en todos los órdenes, incluyendo las cuestiones de género, siendo una necesidad social. En todo caso, hemos de tener en cuenta que el respeto y la valoración inherente a la mirada intercultural es hacia las personas siempre, no necesariamente hacia sus costumbres o conductas, pretendiendo reconocer a la otra u otro y a las culturas, más que describirlas.

- No debe confundirse interculturalidad con folklorismo. No podemos quedarnos en las manifestaciones visibles de las culturas de referencia y juzgar a las personas y los pueblos desde ahí. Al mismo tiempo, debemos tener claro que "las tradiciones sólo merecen ser respetadas en la medida en que son respetables, es decir, en la medida exacta en que respetan los derechos fundamentales de los hombres y las mujeres. Respetar 'tradiciones' o leyes discriminatorias es despreciar a sus víctimas.” (Maalouf, 1999, 116)

- La perspectiva intercultural conlleva la promoción de la construcción de la identidad en libertad y (co)responsabilidad, lo cual implica la asunción de las voces de las personas y colectivos desde su legitimidad existencial y requiere la articulación de políticas de igualdad y políticas de identidad que hagan realidad para ellas un nuevo imperativo categórico como el que nos plantea Boavertura de Sousa Santos (2005, 223): "tenemos derecho a ser iguales cada vez que la diferencia nos inferioriza; tenemos derecho a ser diferentes cuando la igualdad nos descaracteriza."

\section{Monográfico}

- Desde esta óptica, los aprendizajes son medios al servicio de los fines educativos. La perspectiva intercultural aquí defendida pretende establecer un reconocimiento en clave de equidad y justicia que no niegue la multiplicidad de realidades que se encuentran detrás de las personas migrantes. Así, resulta fundamental, en este sentido profundizar en la significatividad experiencial de las actividades educativas que debe concretarse a nivel de proyectos, diseño de investigaciones educativas y espacios de aprendizaje.

- Una actitud intercultural debe tener también como prioridad, evitar o minimizar los prejuicios e incrementar nuestra con(s)ciencia sociopolítica. La mirada intercultural es siempre recíproca e implica, además, tener las más altas expectativas con respecto a las personas y colectivos. Así, la calidad de las relaciones en los espacios de aprendizaje son un referente fundamental y resulta contrario a sus principios toda agrupación homogénea y toda concepción individualista y competitiva del aprendizaje.

- Las miradas, sobre todo, hacen preguntas. La mirada intercultural, insistimos, nos exige el reconocimiento y el valor de la alteridad, nos hace ver el rostro (Lévinas, 1991) de la otra persona y cuestionarnos a nosotras y nosotros mismos.

Además, de esta interpelación axiológica, no olvidemos que parece que dentro de la cartografía de las identidades que se ha configurado en el seno de la globalización neoliberal las personas migrantes no existen más que marginalmente, y lo que no es tenido en cuenta no puede ser objeto de reconocimiento y sujeto de derecho real. Estas personas y colectivos no 


\section{II: Educación y Comunicación para el Desarrollo}

pueden considerarse identidades virtuales, pasivas, en cierto sentido ahistóricas. Las políticas sociales y educativas deben ser ejes para la búsqueda de la igualdad de diferencias en clave de equidad, y no meros instrumentos burocráticos de gestión acrítica; de lo contrario, mejor que, al menos, no se disfracen prácticas vacías con discursos supuestamente interculturales. Como sociedad parece que lo único que queremos son trabajadoras/es y mano de obra barata o pintorescos productos de consumo 'vouyerístico' y explotación, pero la realidad es constituida por personas $\mathrm{y}$, por tanto, también culturas $\mathrm{y}$ visiones del mundo distintas ante las que podemos enriquecernos o bien ignorar o discriminar.

En este sentido, quisiéramos enumerar una serie de propuestas que desde las políticas, el desarrollo comunitario y los procesos educativos, resulta necesario asumir para dar respuesta a la diversidad cultural aludida:

- Las reformas y modificaciones que se proyecten educativamente deben tomar en consideración los requerimientos que se deriven de la presencia de personas migrantes, ya sean niños o niñas, jóvenes o personas adultas, para lo que resulta imprescindible trabajar de manera interdisciplinar y desde la cosmovisiones originarias como referente de proyección, para lo que es necesario disponer de un conocimiento preciso y detallado de las características específicas de estos colectivos, su entorno sociocultural y las circunstancias en las que afrontan su incorporación a los nuevos proyectos de vida.

- El establecimiento de medidas de apoyo reales para garantizar procesos educativos de calidad, preñados de solidaridad y justicia a través de los proyectos, investigaciones e intervenciones realizadas en el contexto concreto.

- La promoción de la elaboración y difusión de reflexiones pedagógicas, experiencias innovadoras y materiales didácticos relevantes que ayuden a la sensibilización y proyección social de los trabajos abordados interculturalmente.

Este modo de analizar la realidad puede y debe tener consecuencias para los sistemas educativos, ya que, partimos del principio histórico-cultural y socioconstructivista del papel absolutamente crucial del lenguaje como principal mediador de la cultura (Vigotsky, 1977), como instrumento que nos ofrece la oportunidad de reconstruir mundos de significados y como elemento de intercambio y enriquecimiento social. Y es que la diversidad cultural de nuestra sociedad, gracias al fenómeno de la inmigración está conllevando también una diversidad lingüística sin parangón histórico y donde hay que conjugar tanto la necesidad del aprendizaje de la lengua del país de acogida como un hecho determinante para la inclusión social de las personas inmigrantes, como la necesidad de valorar las lenguas y culturas maternas de las personas que migran, fomentando las propuestas interculturales bilingües.

Esto, evidentemente, tiene su reflejo en las distintas realidades, sobre todo en dos puntos clave del sistema educativo: la educación obligatoria (fundamentalmente la pública) y la educación de personas adultas. La primera, por acoger cada vez más en sus aulas una mayor diversidad cultural y lingüística que está haciendo emerger situaciones 


\section{1/: Educación intercultural para el desarrollo}

hasta ahora inéditas o no tan visibles en principio, lo que en lugar de tomarlo como situación problemática o molesta, debemos considerarlo como una oportunidad para el enriquecimiento personal y profesional, en la medida de que la búsqueda de respuestas a las necesidades educativas de las niñas, niños y jóvenes provenientes de otras culturas y con lenguas maternas diferentes nos debe ayudar a mejorar como educadoras y educadores, ser más innovadores y transformar nuestras prácticas desde una reconceptualización de las propias teorías que las sustentan, llegando incluso a proponernos e interrogarnos sobre la propia función de la educación pública y su sentido en el marco social y cultural que nos envuelve. Todo ello, sin dejar de tener presente y haciendo visible en todo momento al alumnado como personas con derecho y capacidad para recibir una educación de calidad que actúe de elemento compensador de desigualdades tangibles, que en realidad sólo deben verse como diferencias. En este sentido, a lo mejor es necesario recordarnos permanentemente que la migración, la cultura propia y la educación también son derechos humanos. En ese sentido, Banks (1997), señala la importancia de introducir cambios institucionales en las escuelas que tengan en cuenta el currículum, los recursos educativos, los estilos de enseñanzaaprendizaje, las actitudes, los lenguajes, el programa de orientación, la evaluación educativa, la cultura escolar y el curriculum oculto, en definitiva, que la política educativa de los centros tengan en cuenta las peculiaridades de todo el alumnado.

En cuanto a la educación de personas adultas, la necesidad de las personas migrantes respecto al aprendizaje del territorio de acogida es más patente y posiblemente urgente, ya que a la menor 'permeabilidad lingüística' respecto al aprendizaje de idiomas (capacidad normalmente asociada a la infancia) se le unen una serie de condicionantes para la realización de tareas cotidianas, para el acceso al mundo laboral y por lo tanto a determinados recursos, etc. En este sentido, evidentemente la educación de personas inmigrantes adultas posee una serie de peculiaridades asociadas y que la deben hacer alejarse de la 'perspectiva escolar' (Aguado, 2002), vinculándose con las desarrolladas en los entornos comunitarios. En cualquier caso, existen una serie de estereotipos que se han ido generando alrededor de estas personas y colectivos, de los que debemos alejarnos si verdaderamente optamos por una educación intercultural de las personas y de un desarrollo de las comunidades, como son:

- Las personas migrantes constituyen colectivos heterogéneos culturalmente y el que tengan, como todos, necesidades lingüísticas o sociales no significa que su formación cultural en su lengua de origen no sea adecuada o incluso sistematizada. Hay que desterrar la idea generalizada de que estas personas son mayoritariamente analfabetas y cambiar esta concepción por una mayor comprensión de que la diversidad cultural y sus implicaciones no tiene porqué conllevar carencias formativas o necesidades compensatorias en cuanto a capacidades, así como ser conscientes de que existen más formas de sabiduría que las planteadas desde nuestro etnocentrismo.

- Además, aunque no se sepa leer ni escribir (muchas culturas son eminentemente orales), tenemos también que modificar la idea de que ello significa que no se puede aprender una segunda 


\section{II: Educación y Comunicación para el Desarrollo}

lengua o determinadas habilidades, conceptos o actitudes, sino que, en todo caso, habrá que reconsiderar y contextualizar cuestiones metodológicas y propuestas pedagógicas a las peculiaridades de las personas y sus necesidades.

- El que las necesidades prioritarias de las personas de origen migrante sean a menudo orales y que se parta de temas y léxico de la vida cotidiana, no significa que haya que marginar las reflexiones gramaticales ni hacer menguar las expectativas que respecto al potencial de aprendizaje de estas personas debemos tener.

Creemos necesario optar por aprendizajes experienciales que conlleven a su vez la valoración del fomento de la creatividad y el diálogo intercultural, la recuperación del valor de la tradición oral, la contextualización sociocultural y generacional, el (re) encuentro con el texto y su utilidad social, así como fomentar desde la educación y la enseñanza de la lengua, la lectura crítica del mundo, como diría Freire (1997), que nos pueden ayudar a adquirir los valores interculturales que Fermoso (1997), ha recogido a modo de competencias y habilidades a tener en cuenta en estas relaciones:

- Capacidad para enjuiciar y resolver conflictos interétnicos.

- Aceptación de la posibilidad de diferentes perspectivas culturales.

- Decidida resolución de vivir con quienes pertenecen a otras culturas, aunque sean inmigrantes $y$ minoritarias.

- Aprecio de la propia cultura y de la de los demás.

- Preparación para superar los prejuicios racistas, xenófobos e intolerantes.
- Interés por conocer las costumbres e ideologías de los diversos grupos étnicos.

- Conocimientos de los fenómenos migratorios, los contextos culturales, las diversas formas de expresar los mismos sentimientos en cada cultura.

- La estima por las diversas lenguas maternas de los grupos que la tengan propia, aunque sean minoritarios, a fin de evitar el avasallamiento de una lengua respecto de otra.

- Desarrollo de estas habilidades y competencias, tanto en los miembros del país de origen (emigrados) como en los del de acogida.

Así pues, la educación intercultural pretende conseguir en todas las personas y a través de los procesos educativos, una competencia cultural, que se desarrollen a través de una serie de actitudes y habilidades que nos capaciten para saber convivir con personas culturalmente diferentes, no desiguales, en una sociedad diversa, plural y democrática, que se plasmen en contextos de desarrollo diversos, interconectados, interdependientes y plurales.

\section{Conclusión}

Por todo ello, resulta importante dejar claro que en nuestro tiempo, cultura, sociedad, desarrollo personal y comunitario y educación, son conceptos que se deben encontrar necesariamente interconectados. Así, resulta evidente que no podemos indagar en la cultura(s) sin hacerlo en la red de significados que configuran las relaciones sociales, económicas y políticas en la que se desarrollan y contextualizarla local y globalmente. Nuestras sociedades son multiculturales, pluriétnicas, con múltiples realidades y relaciones desde perspectivas de género, de religión, nacionalismos, 


\section{1/: Educación intercultural para el desarrollo}

lenguas, etc. Pero la realidad es que la política social, y especialmente las políticas educativas, no han apostado realmente por una educación intercultural que, no sólo relacione la educación con las diferencias culturales, sino que establezca ese fenómeno social que conlleva la multiculturalidad como eje principal e imprescindible para construir una nueva forma de ver el desarrollo de las personas y de las colectividades (Ruiz, 2003).

Debemos convencernos de que pluralismo debe significar interdependencia y reciprocidad sin menospreciar la construcción de las identidades culturales, puesto que toda cultura se refiere al ser humano en su dimensión social, optando por una sociedad abierta a la alteridad como eje epistémico y ontológico de cohesión y (re)construcción cultural. Una cultura cerrada, inmovilista, es una cultura moribunda y condenada al aislamiento, el estancamiento y el declive, por lo que, es importante reflejar que una educación intercultural lo que debe hacer es proporcionar conocimientos y enseñar habilidades, estrategias y valores sobre las distintas culturas, presentándolas como significativas en sí misma y elementos de riqueza social, de manera que este aprendizaje le permita a las personas situarse y valorarse dentro de los contextos que les rodean, luchando contra cualquier manifestación de segregación o injusticia.

La mirada intercultural se torna aquí de nuevo fundamental, porque es a través de la misma desde donde podemos asegurar que los grupos y personas desfavorecidas y marginadas dejen de serlo y se sientan partícipes, escuchadas, valoradas y protagonistas de sus propias historias y las que colectivamente necesitamos construir para darle sentido educativo a la propia interculturalidad. Esa misma mirada intercultural debe estar inserta, a su vez, en un proceso de toma de conciencia y emancipación, porque esa pronunciación de la palabra propia requiere un aprendizaje que se da con condiciones para su apropiación. Por ello, es necesario confrontar dialécticamente las políticas y prácticas educativas para que nos conduzcan a una última reflexión en contra de las nuevas formas de totalitarismo emergentes de las premisas neoliberales, las cuales, analizadas desde las políticas educativas, pretenden volver superfluos derechos educativos y culturales de las personas y colectivos, más allá de su 'anticipado' papel en el engranaje instituido, desarrollando prácticas exclusoras para una alta proporción de niños, niñas y jóvenes. Porque la educación intercultural, no es sólo una educación bien intencionada, sino que debe ser considerada una manera de educar que posibilitan, según Astorgano (2000):

- La centralidad de cada ser humano.

- La aceptación de las diferencias como factor de maduración.

- El fomento de una conciencia crítica capaz de analizar y comprender las causas que provocan las migraciones.

- El cultivo metodológico del diálogo.

En cualquier caso, la perspectiva intercultural exige unas connotaciones, (Besalú, 2002; Kincheloe y Steinberg, 1999; Essomba, 1999), que podemos sintetizar en:

- No se dirige a las minorías étnicas o culturales, sino que es una educación para todos y todas. Es una educación para y en la diversidad cultural y no una educación para los culturalmente diferentes. 


\section{II: Educación y Comunicación para el Desarrollo}

- Tiene por objeto promover el reconocimiento de la relatividad de cada persona y la desacralización de todas las culturas.

- Debe promover el conocimiento de la multiculturalidad presente en la sociedad y promover la competencia en múltiples culturas.

- La diferencia debe convertirse en parte del currículum, entendiendo la diversidad cultural en el proceso educativo, no como elemento segregador o diferenciador, sino como elemento enriquecedor, integrador y articulador de la convivencia.

- Se trata de enseñar a los ciudadanos futuros a vivir juntos, en un mismo universo rico y plural. Es una educación para la ciudadanía plena.

- No existe una pedagogía intercultural como tal, sino que la educación intercultural entronca con la mejor tradición pedagógica la que parte y se basa en las necesidades, experiencias e intereses del alumnado y se ocupa de acompañar y potenciar su desarrollo como persona, la que atiende a las personas en su diversidad.

- Evita la separación física de los grupos culturalmente diversos. El primer paso para educar interculturalmente es el contacto físico que ofrece posibilidades reales de mejorar el conocimiento, la comprensión y la estima mutua entre personas pertenecientes a distintas raíces culturales.

- Supone una lucha activa y politizada en contra de las discriminaciones racistas y xenófobas desde un análisis crítico de las estructuras de poder y opresión.

- La mejor educación intercultural es el reconocimiento social y político pleno de las minorías culturales, ya que no podemos responder a los problemas políticos y sociales sólo con soluciones educativas; en este sentido la educación es un acontecimiento político.

Por último, debemos enfatizar que la condición humana básica de la política, es la pluralidad. Sin ella no resulta posible una convivencia real ni un espacio público donde estar-en el mundo junto a los otros y otras. En este sentido, supone una antítesis del pensamiento totalitario y ultraconservador que tanto daño ha hecho y hace a los pueblos. Por ello, debemos insistir en el carácter intercultural que debe estar presente en toda propuesta educativa coherente con una concepción ética y de derechos (y no subordinada a intereses económicos de orden personalista, corporativo o gregario), ya que la desigualdad es producto de injusticias sociales que nos individualizan y descaracterizan, mientras que la diversidad es consustancial al ser humano, siendo la última pretensión, educar a todas y todos para la ciudadanía en el contexto de una sociedad mestiza (Díez, 2012).

\section{Referencias}

Aguado, T. (2002). "El nuevo público de la inmigración y su incidencia en la educación de adultos", en Sanz Fernández, F. (coord.): La educación de personas adultas entre dos siglos: historia pasada y desafíos de futuro. Madrid: UNED.

Astorgano, A. (2000). Educar sin discriminación. XV Semana Monográfica «Aprender para el Futuro». Madrid: Santillana.

Augé, M. (1996). El sentido de los otros. Barcelona: Paidós. 


\section{1/: Educación intercultural para el desarrollo personal y comunitario}

\section{Monográfico}

Banks, S. (1997). Ética y valares en el Trabajo Social. Barcelona: Paidós.

Besalú, X. (2002). "Éxito y fracaso escolar en los alumnos diferentes". Cuadernos de Pedagogía, 315; 72-76.

Bisquerra, R. (2000). Educación emocional y bienestar. Barcelona: Praxis.

Colom, A. J. (2000). Desarrollo sostenible y educación para el desarrollo. Barcelona: Octaedro.

De Sousa Santos, B. (2005). El milenio huérfano. Ensayos para una nueva cultura política. Madrid: Trotta.

Díez, E.J. (2012). Educación intercultural. Málaga: Aljibe.

Essomba, M. A. (coord.) (1999). Construir la escuela intercultural: Reflexiones y propuestas para trabajar la diversidad étnica y cultural. Barcelona: Graó.

Fermoso, P. (1997). "Interculturalismo y educación no formal". En P. Antonio (Coord.): Pedagogía Social. Barcelona: Ariel.

Freire, P. (1997). A la sombra de este árbol. Barcelona: E1 Roure.
Geertz, C. (1991). La interpretación de las culturas. Gedisa: Barcelona.

Kincheloe, J. y Steinberg, L. (1999): Repensar la multiculturalidad. Barcelona: Octaedro.

Lévinas, E. (1991). Ética e infinito. Madrid: Visor. Maalouf, A. (1999). Identidades asesinas. Madrid: Alianza.

Oña, J.M. de (2010). Educación de calle y desarrollo comunitario. Una experiencia educativa en contextos de exclusión. Madrid: Foessa.

Ruiz, C. (2003). Educación intercultural. Una visión crítica de la cultura. Barcelona: Octaedro.

Vigotsky, L. S. (1977). Pensamiento y lenguaje. Buenos Aires: La Pléyade.

Vila Merino, E.S. (2005). Ética, interculturalidad y educación democrática. Hacia una pedagogía de la alteridad. Huelva: Hergué.

Vila Merino, E.S. (2012). "Un juego de espejos. Pensar la diferencia desde la pedagogía intercultural”. Educación XX1, 15(2). 